\title{
IMPROVING DATA COMPRESSION RATIO BY THE USE OF OPTIMALITY OF LZW \& ADAPTIVE HUFFMAN ALGORITHM (OLZWH)
}

\author{
Pooja Jain, Anurag jain and Chetan Agrawal \\ Computer Science Department, Radharaman Institute of Technology and Science, Bhopal
}

\begin{abstract}
There are several data compression Techniques available which are used for Efficient transmission and storage of the data With less memory space. In this paper, we have Proposed a two stage data compression Algorithm that is OLZWH which uses the Optimality of Lempel-Ziv-Welch (OLZW) and Adaptive Huffman Coding. With this proposed Algorithm, the data Compression ratios are Compared with existing Compression Algorithm for different data sizes.
\end{abstract}

\section{KEYWORS}

Optimality of Lempel-Ziv-Welch (OLZW), Adaptive Huffman coding, Compression Ratio.

\section{INTRODUCTION}

As we see any type of data files of any size Character By character, we notice that there are Many recurrence pattern exhibits in it. a data Compression [2] [6] technique takes the advantage Of such repetitive sequence of data in order to Provide a potential cost saving associated with Transmitting less amount of data, reduces storage

Requirement and reduces the probability of transmission errors. A data compression Techniques [4] are divided into two main classes. That are (i) Lossless data Compression and (ii) Lossy data compression In lossless data compression, the compression Process is carried out without loss of data or Information during compression. Examples of such Lossless compression includes A) Statistical data compression Techniques (i) Huffman coding (ii) Run Length Coding B) Dictionary based data compression techniques (i) Lempel- Ziv-Welch (LZW) Huffman coding is a general compression technique in Which data in term of symbol is based on their statistically Occurred frequencies also called probabilities. The Symbols that arise more commonly are assigned a smaller bit whereas the symbol that occur less frequently Are assigned longer bit. in 1977, Abraham Lempel and Jacob Ziv invented a new coding technique for data Compression (LZ77) which uses dictionary for encoding the data Further revised by Welch in 1978 called LZW78.The LZW algorithm is an adaptive dictionary-based Approach that builds a dictionary based on the Document That is being compressed. The LZW begins with An

initial dictionary includes all the symbols in the Alphabet, that builds the Dictionary by adding new phrases in the dictionary. As it come across new symbols in the text which is being compressed. The LZW [1][3][6] compression technique has certain limitations which reduces the compression ratios that are as follows:

DOI : $10.5121 /$ ijit.2015.4102 
(i) The dictionary is initialises for all the characters symbols in at the beginning of the encoding process Some of the dictionary may not be used during encoding of the data files but that unused character symbols occupy some code values that cannot be used for other necessary phrases of dictionary.

(ii) LZW started with 256 encoding code values for 256 character symbols that are 8bit long. The character symbol beyond 256 has to be encoded with 9 bits. Thus, overcome above limitations OLZW [4] [5] compression technique is used.

In OLZW data compression technique, the encoding procedure started with an empty dictionary. The character symbols to be encoded are assigned codes starting from ' 1 ' in the dictionary and the character symbol to be encoded whose index not present in the dictionary is encoded with 8bit ASCII code and inserted into dictionary as a new code. An adaptive Huffman [1][6] coding also called Faller Galler -Knuth algorithm uses the defined word string which determine the Mapping from source message to code word based on a running estimate of the probabilities to the source message. The code is adaptive and changes so as to stay on optimal for the current estimates. An advantage using the Adaptive Huffman coding is that it uses only one pass for compression and provides better performance than static Huffman coding.

\section{OLZWH (Proposed Algorithm)}

In the proposed two stage data compression algorithm (OLZWH), dictionaries formed for input character symbols of OLZW is considered into two segment that are (i) Set of indices, and (ii) Set of ASCII code Proposed algorithm starts with usual OLZW compression process and whenever there is occurrence of ASCII code, Adaptive Huffman algorithm is applied to it. The algorithms for OLZWH and flowchart for compression in Fig.1) \& decompression in Fig(2) shown below

The flow for the same is shown as below-

(i) Compression Algorithm: 


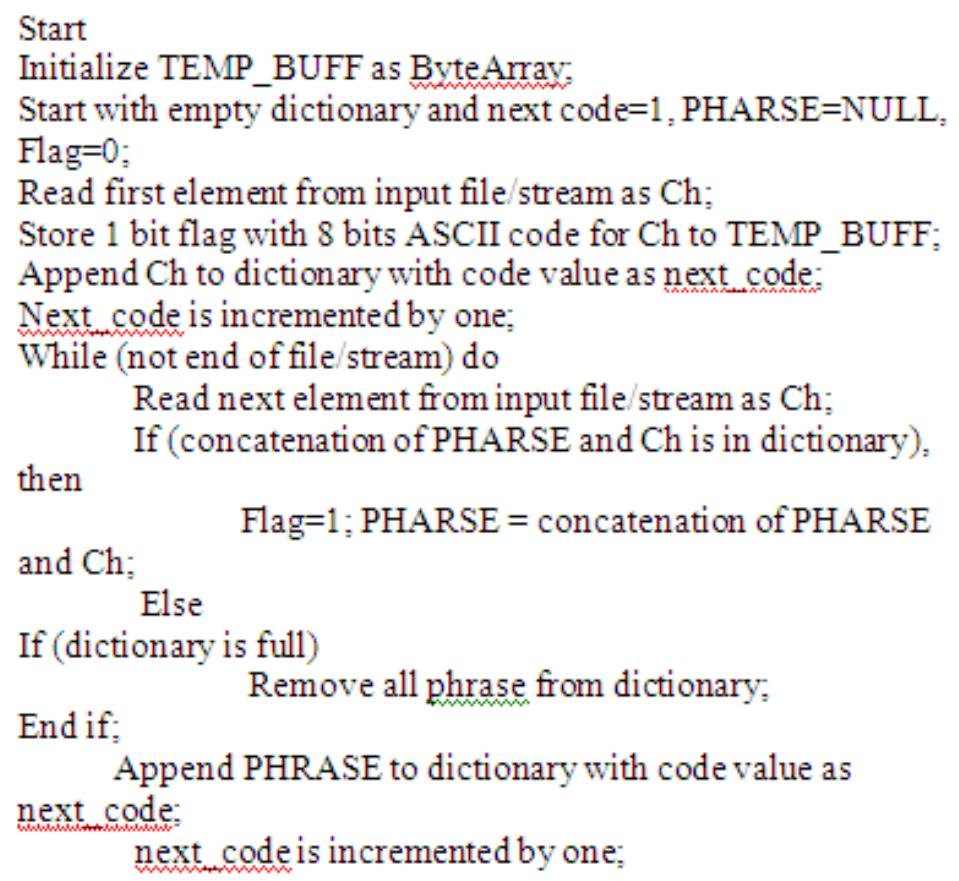


If (Flag=0), then

Store 1 bit flag to output file / stream and

8 bits ASCII code for Ch to TEMP_BUFF;

PHRASE=NULL;

Else

CL=Find_code_length (next_code-1);

Store flag with CL bits code for $\mathrm{Ch}$ from dictionary to

TEMP_BUFF;

If ( $\mathrm{Ch}$ is not in dictionary), then

Flag $=0$;

Store 1 bit flag to output file / stream and

8 bits ASCII code for Ch to TEMP_BUFF

If (dictionary is full)

Remove all phrase from dictionary;

End if;

Append Ch to dictionary with code value as next_code;

next_code is incremented by one;

Else

$\mathrm{PHRASE}=\mathrm{Ch}$;

Flag $=1$;

End if;

End if;

End while;

If (Flag=1), then

$\mathrm{CL}=$ find_code_length (next_code-1);

Store flag with CL bits code for PHRASE from dictionary to output file / stream;

End if;

Initalize COMP_BUFF as ByteArray;

COMP_BUFF=CALL AdaptiveHuffman (TEMP_BUFF)

If (COMP_BUFF.length < TEMP_BUFF) then

Store 1bit flag with COMP_BUFF to the starting of output file / stream;

Else file / stream;

Store 1bit flag with TEMP_BUFF to the starting of output

End if;

END 
International Journal on Information Theory (IJIT),Vol.4, No.1, January 2015

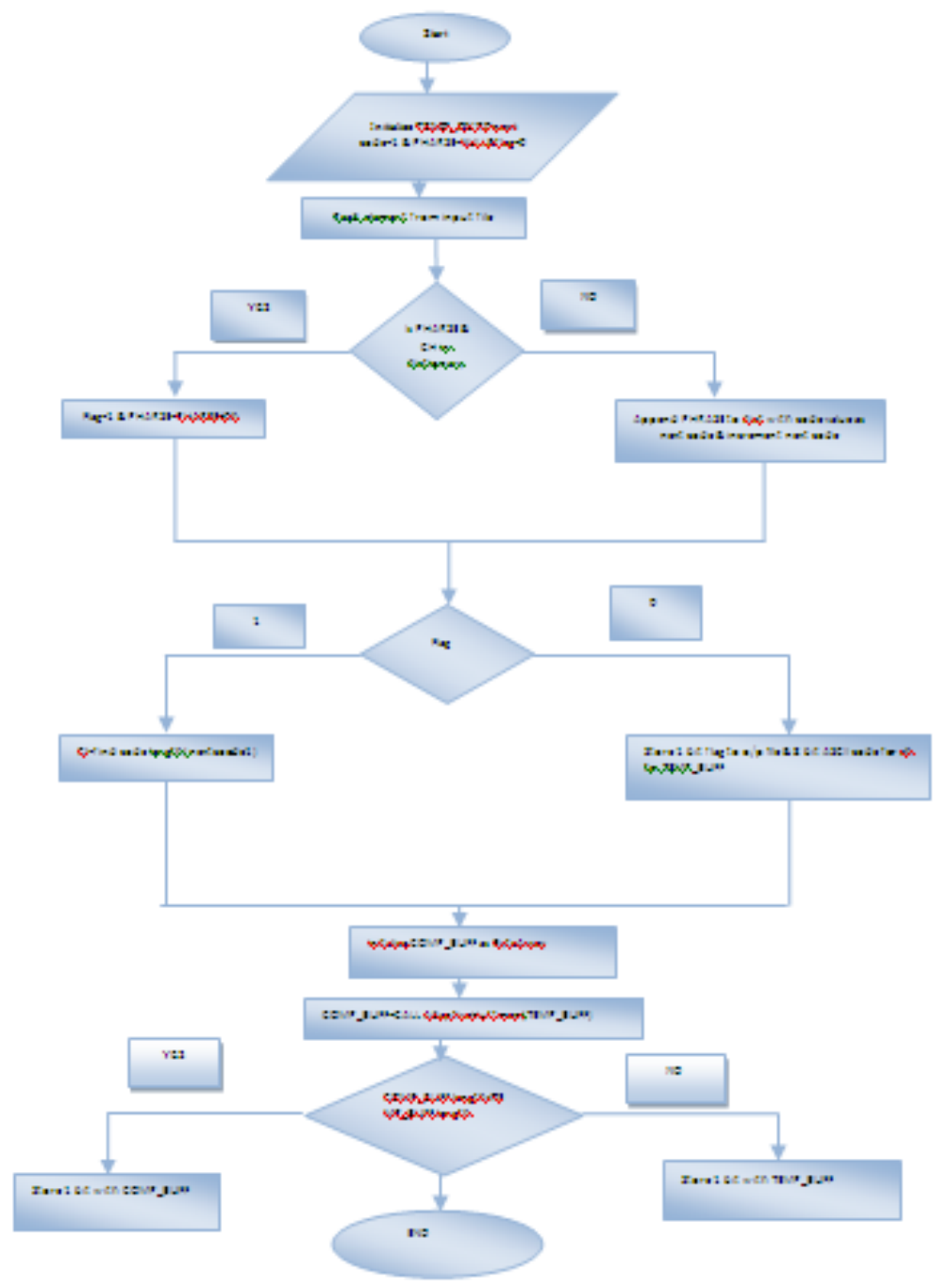

Figure 1 Flow Chart for OLZWH Compression Algorithm 


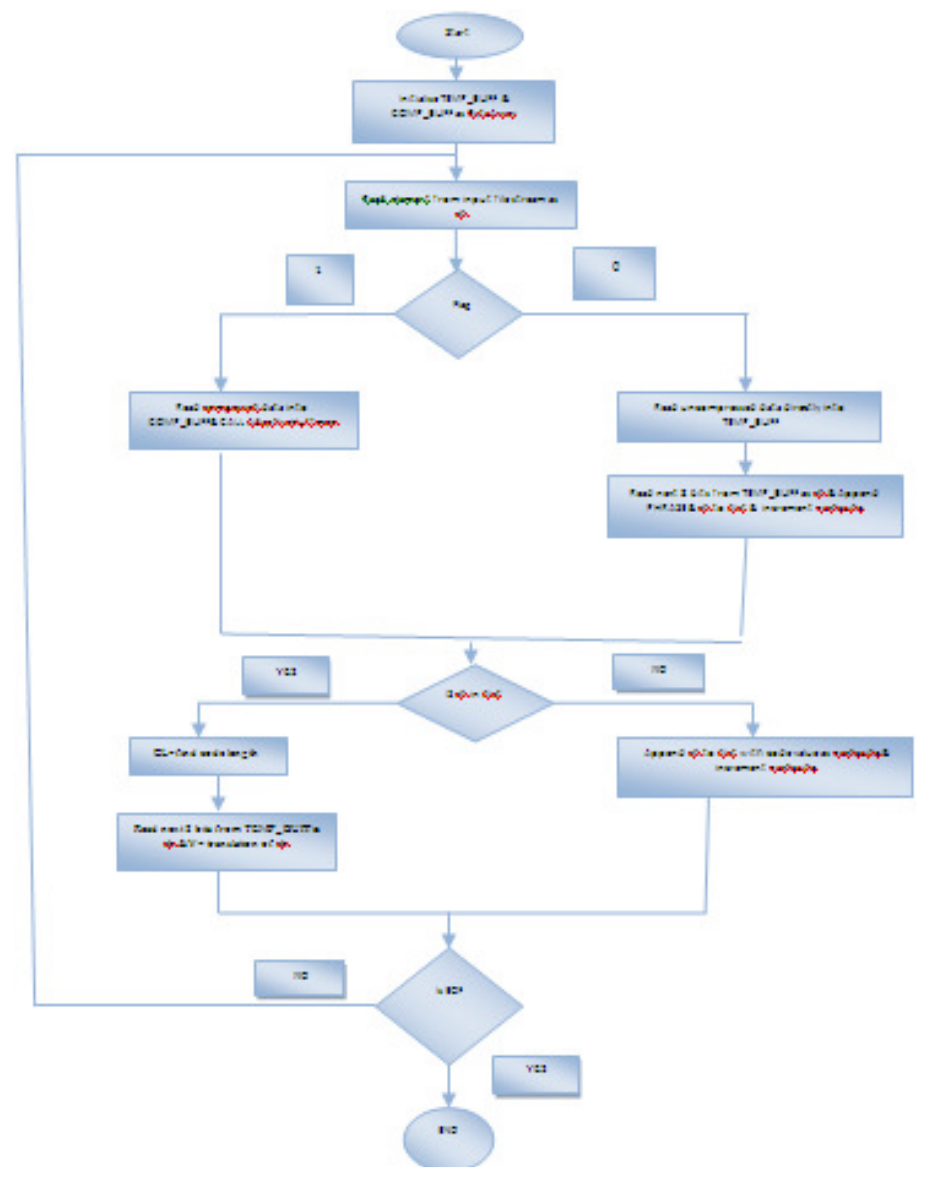

Figure 2 Flow Chart for OLZWH decompression Algorithm

\section{OBSERVATIONS \& RESULTS}

To implement this algorithm and for comparing it with other compression techniques like LZW15, OLZW, we calculated compression ratios in Percentages (\%) for different data files of different sizes are taken. Input data files are shown in table (A) and the chart to show compression ratio in percentage (\%) is shown in (Fig. 3). 
Table - A. Input data files

\begin{tabular}{|l|l|l|l|l|}
\hline File Name & $\begin{array}{l}\text { File Size in } \\
\text { Bytes }\end{array}$ & LZW-15 & OLZW & OLZWH \\
\hline e-mail.doc & 58368 & 51.51 & 58.83 & 60.06 \\
\hline Brain-fingerprint.doc & 81408 & 17.35 & 31.02 & 33.3 \\
\hline Capcha.doc & 219648 & 1.81 & 18.96 & 21.81 \\
\hline Imageprocessing.doc & 411136 & 9.52 & 25.11 & 29.33 \\
\hline 404-1.html & 1358 & 26.8 & 29.23 & 31.15 \\
\hline RSSFeeds.html & 10012 & 56.09 & 62.22 & 66.53 \\
\hline Clickme.html & 41385 & 27.67 & 39.1 & 45.82 \\
\hline $\begin{array}{l}\text { LZWcompression- } \\
\text { Rossett.html }\end{array}$ & 385801 & 56.27 & 64.18 & 77.01 \\
\hline Limiteddic.java & 274 & 16.79 & 18.61 & 20.44 \\
\hline ByteArray.java & 2354 & 35.51 & 45.84 & 47.62 \\
\hline LZW.java & 3706 & 32.68 & 40.66 & 46.41 \\
\hline Compactinputstream.java & 5242 & 30.71 & 39.93 & 48.42 \\
\hline About_continue.help.txt & 1185 & 39.66 & 40.68 & 41.86 \\
\hline Default.help.txt & 2223 & 38.01 & 45.66 & 46.87 \\
\hline About_eventslog.help.txt & 5637 & 40.29 & 48.99 & 54.55 \\
\hline Appv.instlog.txt & 1013458 & 49.31 & 57.97 & 80.3 \\
\hline
\end{tabular}

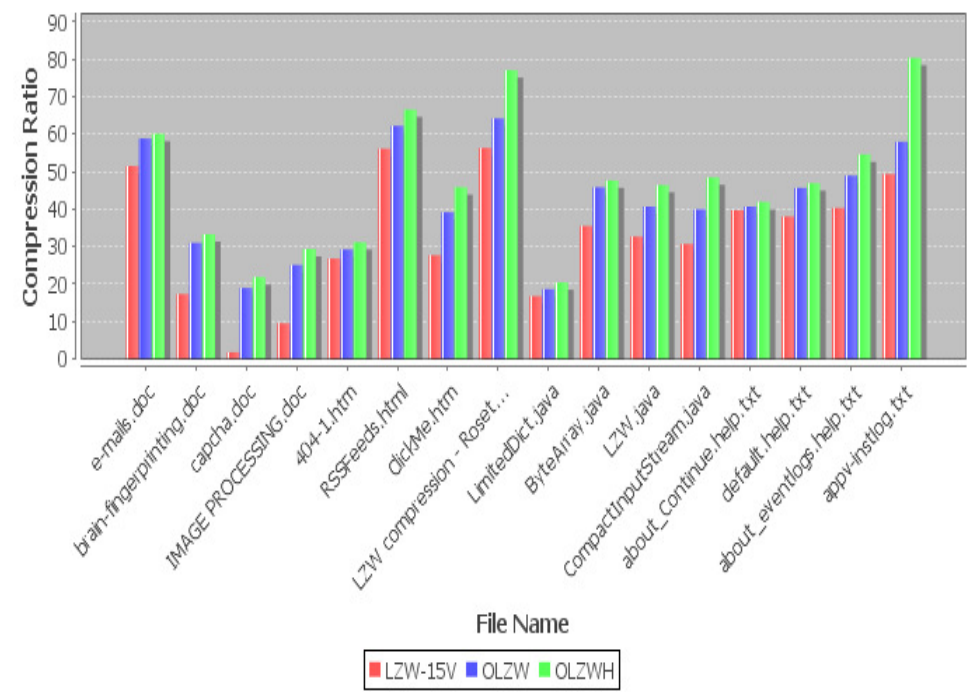

Figure 3. Compression ratio in percentage (\%) 


\section{CONCLUSION}

Thus, the proposed algorithm proves to have better compression ratio for different files as well as different file size than other available data compression techniques. Limitation of OLZW of providing poor compression ratio for larger size is also eliminated. The proposed OLZW technique eliminates some of the problems of the LZW coding and enhances the performance of the same. The proposed technique works very well for particularly small size files most of the time than two versions of LZW (i.e. LZW12 and LZW15V). And the performances of OLZW are not so poor for large size files also. It offers better compressions for large size files than LZW12 most of the time. The compression rates of OLZW for large size files are not as well as LZW15V, but close to it. The proposed technique has a great scope of modifications to make it suitable for large size files also by populating the dictionary with combination of character of phrase instead of the phrase itself and removing phrases not used for longest period of time if the dictionary gets full. It can also be used for image compression.

Dictionary Based Compression Algorithm is found to be more better than LZW in terms of file size reduction. Dictionary Based Compression is better in large size text file and it is similar to LZW Compression file when the file size is small. Dictionary Based Compression algorithm is to search a pattern in the compressed file without getting decompressed to it. Hence reducing the searching time because of being done it in compressed file. So, it would be proved as an optimal searching concept for pattern searching in the compressed text files.

\section{REFERENCES}

[1] Raja P. And Saraswathi,"An effective two stage Text compression \& decompression technique For data compression " International journal of Electronics \& communication engineering (ISSN 09742166) vol.4 no. 29,2011

[2] Ming-Bo lin, jang-feng Lee\& gene Eu jan, "A lossless data compression \& decompression Algorithm and its hardware Architecture"IEEE Transaction on September 2006.

[3] Haroon Altarownesh ,Mohmmad Altarownesh "A data compression technique on text files, A comparison study" International journal of Computer application .(0975-8887) vol.26, No.5,july 2011.

[4] Nishad P.M., Monicka chezian , “ Optimization (Lempel Ziv Welch) algorithm to reduce time Complexity for dictionary creation in encoding \& decoding” AJCSIT (ISSN 2249-5126)

[5] Utpal Nandi, Jyotsna Kumari 'Modified Compression technique based on Optimality of LZW code" Elsevier 2013

[6] MIT 6.02 Draft lecture notes Chapter 3, Compression Algorithm: Huffman \& Lempel Ziv Welch. 